\title{
The effect of influenza vaccine on severity of COVID-19 infection: An original study from Iran
}

\author{
Saeed Kalantari ${ }^{1}$, Afsaneh Sadeghzadeh-Bazargan ${ }^{2}$, Saedeh Ebrahimi ${ }^{1}$, Zeynab Yassin ${ }^{1}$, Seyed Hamid Reza Faiz ${ }^{3}$, \\ Ali Kabir ${ }^{4}$, Amir Baghestani ${ }^{5}$, Farzaneh Mashayekhi ${ }^{5}$, Farah Bokharaei-Salim ${ }^{6}$, Azadeh Goodarzi ${ }^{2} *$ (D) \\ Received:23 Oct 2020 \\ Published: 7 Sep 2021
}

\begin{abstract}
Background: The COVID-19 infection is a novel virus that mainly targets the respiratory system via specific receptors without any coronavirus-targeted therapies. Many efforts have been made to prepare specific vaccines for COVID-19 or use of prefabricated vaccines of other similar viruses, especially severe acute respiratory syndrome (SARS), Middle East respiratory syndrome (MERS), and influenza (flu). We aimed to evaluate the effects of previous flu vaccine injection on severity of incoming COVID-19 infection.

Methods: We conducted a large cross-sectional study of 529 hospitalized Iranian COVID patients to evaluate the severity of disease courses in patients with or without previous flu vaccination history using some main factors like length of hospitalization, need for the intensive care unit (ICU) admission and length of stay in the ICU for comparison between COVID-19 infected patients with or without flu vaccination history. For the quantitative data, we used independent-samples $t$ and Mann-Whitney tests. The qualitative data were calculated using the Fisher exact and chi-square tests in IBM SPSS Statistics version 22 (SPSS Inc) andl P value $<0.05$ was considered statistically significant.

Results: There were no significant differences in the demographic data of patients, disease, and severity-related parameters between the 2 groups. It means that there were not any significant differences between patients with and without history of flu vaccination regarding mean days of hospitalization, percentage of needing to be admitted to the ICU, days being admitted to the ICU ( $8.44 \pm 6.36 \mathrm{vs}$ $7.94 \pm 8.57 ; 17 \%$ vs $11.5 \%$; and $1.17 \pm 3.09$ vs $0.92 \pm 3.04$, retrospectively) ( $\mathrm{p}=0.883,0.235$, and 0.809 , respectively).

In the laboratory tests, in comparison between patients with and without history of previous flu vaccination, only lymphocytes count in the vaccine positive group was higher than the vaccine negative group $(20.82 \pm 11.23$ vs 18.04 \pm 9.71$)(\mathrm{p}=0.067)$ and creatine phosphokinase (CPK) levels were higher in the vaccine negative group $(146.57 \pm 109.72$ vs $214.15 \pm 332.06)(p=0.006)$.

Conclusion: We did not find any association between flu vaccination and decrease in disease severity in our patients. It seems that patients with previous history of flu vaccination may experience less laboratory abnormalities in some parameters that could be interpreted in favor of lower overall inflammation; however, this study cannot answer this definitely because of its design. As we collected retrospective data from only alive discharged patients and had no healthy control group, we could not discuss the probable effect of the vaccine on the mortality rate or its probable protective role against the infection. We need more well-designed controlled studies with different populations in different geographic areas to address the controversies.
\end{abstract}

Keywords: Corona, COVID-19, SARS-CoV-2, Influenza Vaccine, Flu, Vaccine, Vaccination, Severity, Outcome

Conflicts of Interest: None declared

Funding: None

${ }^{*}$ This work has been published underCC BY-NC-SA 1.0 license.

Copyright $\odot$ Iran University of Medical Sciences

Corresponding author: Dr Azadeh Goodarzi, goodarzi.a@iums.ac.ir

1. Department of Infectious Disease, Antimicrobial Resistance Research Center, Rasool Akram Medical Complex, Iran University of Medical Sciences, Tehran, Iran

2. Department of Dermatology, Rasool Akram Medical Complex, Iran University of Medical Sciences, Tehran, Iran

3. Minimally Invasive Surgery Research Center, Iran University of Medical Sciences, Tehran, Iran

4. Department of Epidemiology, Minimally Invasive Surgery Research Center, Iran University of Medical Sciences, Tehran, Iran

5. Department of General Medicine, Rasool Akram Medical Complex, Iran University of Medical Sciences, Tehran, Iran

6. Department of Virology, School of Medicine, Iran University of Medical Sciences, Tehran, Iran $\uparrow$ What is "already known” in this topic:

Many efforts have been made to prepare specific vaccines for COVID-19 or use of prefabricated vaccines of other similar viruses, especially severe acute respiratory syndrome (SARS), Middle East respiratory syndrome (MERS), and influenza (flu), since they may decrease infection rate and disease severity or improve final outcomes while being infected.

\section{$\rightarrow$ What this article adds:}

There is no association between influenza vaccination and decrease in disease severity in our patients. However, we collected retrospective data from only alive discharged patients and had no healthy control group. More well-designed controlled studies with different populations in different geographic areas are needed to address the controversies. 
Cite this article as: Kalantari S, Sadeghzadeh-Bazargan A, Ebrahimi S, Yassin Z, Faiz SHR, Kabir A, Baghestani A, Mashayekhi F, Bokharaei-Salim F, Goodarzi A. The effect of influenza vaccine on severity of COVID-19 infection: An original study from Iran.Med J Islam Repub Iran. 2021(7 Sep);35:114. https://doi.org/10.47176/mjiri.35.114

\section{Introduction}

The COVID-19 infection is a novel virus that mainly targets respiratory system via specific receptors without any corona virus- targeted therapies; there are many other respiratory-associated viruses (like the influenza virus), which result in some similar clinical features (1) and there are some experiences about their vaccination, such as vaccine efficacy and its protective and therapeutic roles (eg, reduction of disease severity) (2). In addition to lack of virus-specific therapies, nowadays, COVID-19 vaccination is limited to some clinical trials and there is not any standard vaccination product and protocol yet, so one of management strategies in this pandemic is focusing on protective, therapeutic, and immune enhancing potentials of available vaccines of other respiratory-associated virus that may be beneficial for COVID-19 and may reduce severity of infection (3-5).

Based on the current literature review, it is found that there are many studies about COVID-19 vaccination also about the effects of the flu vaccine on various aspects of COVID-19; and logically there may be some controversies in these entities that need more evaluations in different populations in different geographic areas. In a systematic review about therapy and vaccination of COVID-19, the authors found that there were 5 vaccines under investigation in phase 1 trials for the corona virus and other similar viruses also there were 2 other phase 3 trials on BCG (Bacillus Calmette-Guérin) vaccine, so it seems we need an approved vaccination for breaking the chain of COVID-19 spreading (6). In another systematic review, it has been discussed that although phase 1 trials on DNAbased virus vaccines are available, vaccines which target other similar viruses like MERS-CoV-SARS- CoV could be effective options in this pandemic (pang). In May 2020, 1 other systematic review reported 10 human clinical trials on vaccination for COVID-19, 5 in phase 1, 2 in phase 2, and 3 in phase 3 (7). In a study that evaluated the effects of previous flu vaccine on COVID-19-asociated outcome, means mortality, the authors found some positive protective effects of vaccine on COVID-19, especially in elderly population (8). In another study no effect of the flu vaccine was found on other respiratory-associated viruses like corona-viruses also the vaccine does not increase the risk of latter mentioned viruses (9).

We supposed that the flu vaccine may decrease the severity of COVID-19 infection in hospitalized patients with respect to duration of hospitalization and their need to treatment change or ICU admission, as it seems that the flu vaccine by increasing immunogenicity toward respiratory-associated viruses, may decrease the severity of disorder. Also, for a more more complete discussion about this hypothesis, we need more well-designed casecontrolled studies to find the protective effect of the flu vaccine as well. In this large cross-sectional study from Iran, we tried to evaluate the effects of previous flu vaccine injection on the severity of incoming COVID-19 in- fection.

\section{Methods}

This analytical cross-sectional study was conducted on 529 Iranian patients affected by COVID-19, between March to May, 2020, who were hospitalized in RasoolAkram Medical Complex affiliated to Iran University of Medical Sciences, Tehran, Iran. Their diagnoses were approved by a positive nasopharynx reverse transcription polymerase chain reaction (RT-PCR) test or in the case of negative PCR; based on very suggestive computed tomography imaging for COVID-19 scored by the CO-RADS classification system (10). From 529 patients, 59 cases $(11.15 \%)$ had a positive history of the flu vaccine injection. We tried to compare the COVID-19 diseases severity in patients with or without vaccine history. The main outcomes of this study for comparison between 2 groups (which were considered indirectly to be associated with the overall severity score of the disease) were the mean days of hospitalization, the necessity for ICU admission, the mean days of staying in the ICU and the need to take second-line therapeutic options. For both groups we assessed demographic data of the disease and the patients, laboratory tests, and treatment protocols completely. The continuous variables were presented as mean and SD and for quantitative data we used independent-samples $t$ test and Mann-Whitney test. The qualitative data were calculated using the Fisher exact and chi-square tests in IBM SPSS Statistics version 22 (IBM), and $\mathrm{P}$ value $<.05$ was considered statistically significant. The ethical code of the large cohort study of RasoolAkram Medical Complex from which these data were extracted was IR.IUMS.REC.1399.759.

\section{Results}

Demographic data of the study participates are presented in Table 1. There were no significant differences in the demographic data of patients, disease, and severity-related parameters between the 2 groups and only the chest discomfort was significantly more prevalent in the influenzanegative vaccine group $(42.4 \%$ vs $30 \%)(\mathrm{p}=0.041)$ (Table $1)$. In the laboratory tests, only the lymphocytes count in the vaccine positive group was higher than the vaccine negative group $(\mathrm{p}=0.067)$ and CPK levels were higher in the vaccine negative group $(\mathrm{p}=0.006)$ (Table 2$)$. The length of hospital stay for the influenza-positive vaccine group was $8.44 \pm 6.36$ days and for the influenza negative vaccine group was $7.94 \pm 8.57$ days $(\mathrm{p}=0.924)$. The need to ICU admission in the influenza-positive vaccine group was $16.9 \%$, and in the influenza- negative vaccine group was $11.5 \%(p=0.235)$, and the length of ICU admission for the influenza-positive vaccine group was $1.17 \pm 3.09$ days, and for the influenza negative vaccine group was $0.92 \pm 3.04$ days $(\mathrm{p}=0.809)$. The need to second-line thera- 
C Kalantari at al

Table 1. Disease and patients' characteristics in patients with positive and negative history of influenza vaccination

\begin{tabular}{|c|c|c|c|}
\hline Patient or disease chactristics & $\begin{array}{l}\text { Influanza-Vaccine (positive) } \\
\qquad(\mathrm{n}=59)\end{array}$ & $\begin{array}{l}\text { Influanza-Vaccine (negative) } \\
\qquad(\mathrm{n}=470)\end{array}$ & $\mathrm{p}$ \\
\hline Gender (male) $n \%$ & $32(54.2)$ & $196(41.7)$ & 0.578 \\
\hline Age (year) (Mean \pm SD) & $57.78 \pm 6.36$ & $59.11 \pm 16.21$ & 0.883 \\
\hline Hospitalization Days & $8.44 \pm 6.36$ & $7.94 \pm 8.57$ & 0.924 \\
\hline ICU Days & $1.17 \pm 3.09$ & $0.92 \pm 3.04$ & 0.809 \\
\hline IVIG (positive) & $7(11.9)$ & $40(8.5)$ & 0.463 \\
\hline ICU Admission & $10(16.9)$ & $54(11.5)$ & 0.235 \\
\hline PCR (positive) & $6(10.2)$ & $47(10.0)$ & 1.000 \\
\hline chills (yes) & $35(59.3)$ & $307(65.3)$ & 0.560 \\
\hline Fever (positive) & $38(64.4)$ & $295(62.80)$ & 0.887 \\
\hline Fever length & $2.53 \pm 3.36$ & $2.37 \pm 3.40$ & 0.746 \\
\hline Dyspnea (positive) & $43(72.9)$ & $309(65.7)$ & 0.180 \\
\hline Fatigue (positive) & $48(72.9)$ & $335(71.3)$ & 0.850 \\
\hline Anorexia (positive) & $44(74.6)$ & $279(59.4)$ & 0.120 \\
\hline Body pain (positive) & $35(59.3)$ & $279(59.4)$ & 0.886 \\
\hline Diarrhea history (positive) & $16(27.1)$ & $80(17)$ & 0.420 \\
\hline New Diarrhea (positive) & $5(8.5)$ & $47(10)$ & 1.000 \\
\hline Sore through (positive) & $16(27.1)$ & $99(21.1)$ & 0.230 \\
\hline Nausea and Vomiting (positive) & $22(37.3)$ & $164(34.9)$ & 0.773 \\
\hline Sputum (positive) & $22(37.3)$ & $121(25.7)$ & 0.570 \\
\hline Chest discomfort (positive) & $25(42.4)$ & $141(30)$ & 0.041 \\
\hline Headache (positive) & $18(30.5)$ & $161(30)$ & 0.881 \\
\hline Vertigo (positive) & $16(27.1)$ & $109(23.2)$ & 0.321 \\
\hline Delusion (positive) & $5(8.5)$ & $50(10.6)$ & 1.000 \\
\hline Seizure (positive) & $2(3.4)$ & $8(1.7)$ & 0.285 \\
\hline LOC (positive) & $8(13.6)$ & $80(17)$ & 0.706 \\
\hline Anosmia/hyposmia(positive) & $17(28.8)$ & $93(19.8)$ & 0.780 \\
\hline Dsgeusia (negative) & $11(18.6)$ & $92(19.6)$ & 1.000 \\
\hline Heart Disease (positive) & $15(25.4)$ & $116(24.7)$ & 0.748 \\
\hline Lung Disease (positive) & $10(16.9)$ & $46(9.8)$ & 0.113 \\
\hline Kidney Disease (positive) & $8(13.6)$ & $44(9.4)$ & 0.233 \\
\hline Dialyzed (positive) & $1(1.7)$ & $15(3.2)$ & 1.000 \\
\hline Immunodeficiency (positive) & $4(6.8)$ & $4(0.9)$ & 1.000 \\
\hline Diabetes Mellitus (positive) & $19(32.2)$ & $145(30.9)$ & 0.762 \\
\hline Hypertension (positive) & $21(35.6)$ & $132(28.1)$ & 0.217 \\
\hline Malignancy (positive) & $5(8.5)$ & $21(4.5)$ & 0.181 \\
\hline Tuberculosis (negative) & $1(1.7)$ & $7(1.5)$ & 1.000 \\
\hline Laboratory tests & $\begin{array}{c}\text { Influanza-Vaccine (positive) } \\
(\mathrm{n}=59)\end{array}$ & $\begin{array}{c}\text { Influanza-Vaccine (negative) } \\
(\mathrm{n}=470)\end{array}$ & $\overline{\mathrm{p}}$ \\
\hline WBC & $8.48 \pm 5.20$ & $7.47 \pm 4.39$ & 0.111 \\
\hline Diff_segment & $57.34 \pm 30$ & $57.61 \pm 32.40$ & 0.951 \\
\hline Diff_lymphocyte & $20.82 \pm 11.23$ & $18.04 \pm 9.71$ & 0.067 \\
\hline $\mathrm{ESR}^{-}$ & $45.29 \pm 23.56$ & $48.86 \pm 26.65$ & 0.367 \\
\hline CRP & $10.98 \pm 17.72$ & $8.05 \pm 15.28$ & 0.229 \\
\hline $\mathrm{Cr}$ & $1.18 \pm 0.66$ & $1.14 \pm 0.61$ & 0.697 \\
\hline AST & $34.70 \pm 16.93$ & $39.74 \pm 28.85$ & 0.223 \\
\hline ALT & $24.20 \pm 18.77$ & $27.01 \pm 32.13$ & 0.512 \\
\hline LDH & $571 \pm 241.93$ & $615 \pm 258$ & 0.248 \\
\hline CPK & $146.57 \pm 109.72$ & $214.15 \pm 332.06$ & 0.006 \\
\hline \multicolumn{4}{|c|}{ Table3.Initial therapies during hospitalization in patients with positive and negative history of influenza vaccination } \\
\hline supplementary treatments & $\begin{array}{l}\text { Influanza-Vaccine (positive) } \\
\qquad(\mathrm{n}=59)\end{array}$ & $\begin{array}{l}\text { Influanza-Vaccine (negative) } \\
(\mathrm{n}=470)\end{array}$ & $\mathrm{p}$ \\
\hline Azithromycin & $22(37.3 \%)$ & $158(33.6 \%)$ & 0.576 \\
\hline Heparin (1=yes) & $44(74.6 \%)$ & $348(74 \%)$ & 0.930 \\
\hline Lopinavir_and ritonavir & $40(67.8 \%)$ & $326(69.4 \%)$ & 0.807 \\
\hline Linezolid & $18(30.5 \%)$ & $97(20.6 \%)$ & 0.123 \\
\hline Hydroxyl chloroquin & $53(89.8 \%)$ & $413(87.9 \%)$ & 0.662 \\
\hline
\end{tabular}

py means intravenous immunoglobin (IVIG) therapy in influenza-positive vaccine group was $11.9 \%$ and in the influenza-negative vaccine group was $8.5 \% \quad(p=0.235)$ (Table 1). In Table 3, you can see the initial therapies of both groups that were not statistically different.

\section{Discussion}

COVID-19 vaccination encompasses DNArecombinant, mRNA-based, inactivated whole virus, and live attenuated virus vaccines. There are only few vaccines that progressed to release the initial results, including MERS-CoV and SARS-CoV vaccine, which have shown positive outcomes that resulted in entrance to trial phase 2 . As the SARS virus has genetic similarity of about $79 \%$ to the novel corona virus, it is expected that this vaccine has a promising effect on COVID-19 (7). It is proposed that in COVID-19 pandemic, especially in elderly people, one of main pathologic events is the downregula- 
tion of the innate immune system, and as the live attenuated virus vaccines have proved to have positive effects on activity of this system, the vaccines (like influenza vaccine) could enhance the immune system function, and logically result in favorable achievements in final outcomes of the new corona virus (8).

With respect to COVID-19 vaccination, there are many clinical trials in different phases, some of which showed promising outcomes, although more evaluations and phase progression of clinical trials are necessary for more definite results $(3,6,7)$. In a recent systematic review published May, 2020, a total of 10 human clinical trials were conducted on COVID vaccination, from which 7 studies focused on SARS-CoV-2 S-protein and 3 on the protective effects of the BCG vaccine based on lymphocyte activation theory. Immune-mediated preventive and therapeutic strategies are the hot topics in this pandemic area, especially via nanotechnology, which may enhance the efficacy and reduce the side effects of different modalities like vaccines (11).

With respect to influenza vaccination in COVID pandemic, there are some articles with various designs and purposes. In a study written by Grech V and Borg M, the authors said that although coinfection of COVID-19 and influenza does not seem to worsen patients' outcome in ongoing cold seasons, if we have a world-based vaccination we may expect to decrease the burden of the flu and more capacity to manage COVID patients (12). Grohskopf et al study also confirmed this theory (13).

In their study, Skowronski et al found no protective effect of influenza vaccine on respiratory associated viruses like corona viruses; also, they found that the vaccine did not increase the risk of latter mentioned viruses (9).

In a study on previous influenza vaccine effect on COVID-19-asociated outcome, the authors found some positive protective effects of vaccine on COVID patients, especially on mortality rates of the elderly (8). However, in the present study, as we did not have a control group, we could not compare our results with the mentioned study due to not assessing the protective effect of vaccine. Although in our population, we did not find any severity reduction by previous influenza vaccine injection.

In Fink et al study from Brazil on 92,664 COVID patients, the authors evaluated the effect of influenza vaccine on the severity of the disease. They found about $8 \%$ lower odds of needing ICU admission, an $18 \%$ decrease in need for invasive respiratory support and a $17 \%$ decrease in mortality rate. Although these difference percentages are not high, considering the pandemic encountered, these differences are responsible for a large number of human beings, which is of great importance in life saving and financial issues (14). Unlike Fink et al study, we did not see any decrease of disease severity in our study, which could be because of our smaller sample size, lack of control group, various intervals of vaccine injection, and COVID-19 infection, and lack of assessment of mortality rate due to the retrospective design of our study. We only assessed duration of hospital stay, necessity to ICU admission, mean days of ICU admission, and need to IVIG therapy as severity-related parameters of this study.
The authors of this study have worked on various aspects of COVID-19 (15-28), and have tried to report the results of this study to evaluate the theory of beneficial effects of influenza vaccine-induced immunogenicity toward COVID-19; however, they did not find any significant association in this study.

\section{Study Limitations}

In this study, we did not have a control group to evaluate the protective effect of the vaccine. Also, we did not consider mortality in our outcomes, as we retrospectively collected the data of vaccination of our discharged alive patients and we did not gather the data of patients who died during hospitalization. We did not ask about type of the flu vaccine (such as trivalent or quadrivalent), which we suggest asking in future studies so that researchers may find any association between type of vaccine and its protective or immune-enhancing effect against COVID19. In our study, the clinical and imaging severity scores of patients were not calculated based on predefined scores, as during the data gathering of this study, these scores were not as popular as they are now, and the main outcomes of this study for comparison between the 2 groups (which were considered indirectly to be associated with the overall severity score of the disease) were the mean days of hospitalization, the necessity for ICU admission, the mean days of staying in ICU, and the need to take second-line therapeutic options.

\section{Conclusion}

Although we did not find any association between the influenza vaccination and decrease of disease severity in our patients, for more definite comments about the protective and immune enhancing roles of the influenza vaccine in COVID-19 pandemic, we need more well-designed controlled studies of different populations in different geographic areas to address the controversies.

\section{Acknowledgment}

The authors would like to thank Rasool Akram Medical Complex Clinical research development Center (RCRDC) for its technical and editorial assists.

\section{Conflict of Interests}

The authors declare that they have no competing interests.

\section{References}

1. Li X, Geng M, Peng Y, Meng L, Lu S. Molecular immune pathogenesis and diagnosis of COVID-19. J Pharm Anal. April 2020;10(2):102-108

2. Lurie N, Saville M, Hatchett R, Halton J. Developing Covid-19 Vaccines at Pandemic Speed. N Engl J Med. 2020;382:1969-1973.

3. Pang J, Wang MX, Ang IYH, Tan SHX, Lewis RF, ChenJIP,et al. Potential Rapid Diagnostics, Vaccine and Therapeutics for 2019 Novel Coronavirus (2019-nCoV): A Systematic Review. J Clin Med. 2020;9(3):623.

4. Osterholm MT, Kelley NS, Sommer A, Belongia EA. Efficacy and effectiveness of influenza vaccines: a systematic review and metaanalysis [published correction appears in Lancet Infect Dis. 2012 Sep;12(9):655]. Lancet Infect Dis. 2012;12(1):36-44.

5. Lansburya PE, SmithaSh, Beyer W, Karamehic E, PasicJuhas E, Hana Sikira H,et al. Effectiveness of 2009 pandemic 
influenza $\mathrm{A}(\mathrm{H} 1 \mathrm{~N} 1)$ vaccines: A systematic review and meta-analysis. Vaccine.2017;35:1996-2006

6. Bhagavathula AS, Aldhaleei WA, Rovetta A, Rahmani J. Vaccines and Drug Therapeutics to Lock Down Novel Coronavirus Disease 2019 (COVID-19): A Systematic Review of Clinical Trial. Cureus. 2020 May;12(5):e8342.

7. Checcucci E, Piramide F, Pecoraro A, Amparore D, Campi R, Fiori C,et al. The vaccine journey for COVID-19: a comprehensive systematic review of current clinical trials in humans. Panminerva Med. 2020 May 26.

8. Zanettini C, Omar M, Dinalankara W, Imada EL, Colantuoni E, Parmigiani G,et al. Influenza Vaccination and COVID19 Mortality in the USA. medRxiv. June 262020

9. Skowronski DM, Zou M, Clarke Q, Chambers C, Dickinson JA, Sabaiduc S,et al. Influenza vaccine does not increase the risk of coronavirus or other non-influenza respiratory viruses: retrospective analysis from Canada, 2010-11 to 2016-17. Clin Infect Dis. 2020 May 22.

10. Prokop M, Everdingen W, Vellinga T, Quarles van Ufford H, Stöger L,et al. CO-RADS: A Categorical CT Assessment Scheme for Patients Suspected of Having COVID-19-Definition and Evaluation Radiology. 2020 296:2:E97-E104.

11. Florindo HF, Kleiner R, Vaskovich-Koubi D, Acúrcio RC, Carreira $\mathrm{B}$, Yeini E, et al. Immune-mediated approaches against COVID-19. Nature Nanotechnol. August 2020;15:630-645.

12. Grech V, Borg M. Influenza vaccination in the COVID-19 era. Early Hum Dev. 2020 Sep;148:105116.

13. Grohskopf LA, Liburd LC, Redfield RR. Addressing Influenza Vaccination Disparities During the COVID-19 Pandemic. JAMA. 2020 Aug 20.

14. FinkG, Orlova-Fink N, Schindler T, Grisi S, Ferrer AP, Daubenberger $\mathrm{C}$,et al. Inactivated trivalent influenza vaccine is associated with lowermortality among Covid-19 patients in Brazil. medRxiv preprint doi: https://doi.org/10.1101/2020.06.29.20142505.

15. Seirafianpour F, Mozafarpoor S, Fattahi N, Sadeghzadeh-Bazargan A, Hanifiha M, Goodarzi A. Treatment of COVID-19 with pentoxifylline: Could it be a potential adjuvant therapy? [published online ahead of print, 2020 May 30]. Dermatol Ther. 2020;e13733.

16. Seirafianpour F, Sodagar S, Pour Mohammad A, Panahi P, Mozafarpoor S, Almasi S,et al. Cutaneous manifestations and considerations in COVID-19 pandemic: A systematic review. Dermatol Ther. 2020;e13986.

17. Nobari NN, Goodarzi A. Patients with specific skin disorders who are affected by COVID-19: What do experiences say about management strategies? A systematic review. Dermatol Ther. 2020;e13867.

18. Mohamadi MM, Goodarzi A, Aryannejad A, Fattahi N, AlizadehKhoei M, Miri S, et al . Geriatric challenges in the new coronavirus disease-19 (COVID-19) pandemic: A systematic review. Med J Islam Repub Iran. 2020;34(1):841-848.

19. Faiz SHR, Riahi T, Rahimzadeh P, Nikoubakht N. Commentary: Remote electronic consultation for COVID-19 patients in teaching hospitals in Tehran, Iran. Med J Islam Repub Iran. 2020;34(1):217218.

20. Najar Nobari N, Seirafianpour F, Dodangeh M, SadeghzadehBazargan A, Behrangi E, Mozafarpoor S, et al. A systematic review of the histopathologic survey on skin biopsies in patients with Corona Virus Disease 2019 (COVID-19) who developed virus or drug-related mucocutaneous manifestations. Exp Dermatol. 2021 May 11.

21. Sadeghzadeh-Bazargan A, Rezai M, Najar Nobari N, Mozafarpoor S, Goodarzi A. Skin manifestations as potential symptoms of diffuse vascular injury in critical COVID-19 patients. J Cutan Pathol. doi: 10.1111/cup.14059. Online ahead of print.

22. Atefi NS, Behrangi E, Mozafarpoor S, Seirafianpour F, Peighambari S, Goodarzi A. N-acetylcysteine and coronavirus disease 2019: May it work as a beneficial preventive and adjuvant therapy? A comprehensive review study. J Res Med Sci. 2020;25:109.

23. Sadeghzadeh-Bazargan A, Behrangi E, Goodarzi A. Systemic retinoids in the COVID-19 era - are they helpful, safe, or harmful? a comprehensive systematized review study. Iran J Dermatol. 2020;23(Supp.1):S9-12.

24. Sadeghzadeh-Bazargan A, Behrangi E, Goodarzi A. Cytokine storm and probable role of immunoregulatorydrugs in COVID-19: A comprehensive review study. Iran J Dermatol. 2020;23(Supp.1):S1318.
25. Najar Nobari N, Seirafianpour F, Mashayekhi F, Goodarzi A. A systematic review on treatment-related mucocutaneous reactions inCOVID-19patients. Dermatol Ther. 2020:e14662.

26. Mohamadi M, Fattahi N, Goodarzi A, Alizadeh-Khoei M, MiriSh, Hekmat H, et al. A Comprehensive Review on COVID-19 Infection and Comorbidities of Various Organs. Acta Med Iranic. 2021;59(1):414

27. Najar Nobari N, Montazer F, Seirafianpour F, Nikkhah F, Aryanian Z, Goodarzi A. Histopathologic Changes and Cellular Events of Organs Systems in COVID-19. J Cell Mol Anesth. 2021;6(1):81-88.

28. Kooranifar S, Sadeghipour A, Riahi T, Goodarzi A, Tabrizi S, Davoody N. Histopathologic survey on lung necropsy specimens of 15 patients who died from COVID-19: A large study from Iran with a high rate of anthracosis. Med J Islam Repub Iran. 2021 May;35:63. 